\title{
A study of views of spiritual care among junior doctors at a district general hospital
}

\author{
Authors: Dennis Poon, Wendy Yap, Nor Faizul Ahmad, Jia-Wei Tang, David Knight and Aditya Mandal
}

\section{Introduction}

Patients with advanced cancers are often admitted to hospitals for control of symptoms such as pain, nausea and vomiting. Exploring and addressing patients' spiritual needs while they are inpatients is recognised as an essential part of comprehensive palliative care. Studies show that addressing patients' religious and spiritual needs has a positive impact on their spiritual wellbeing and quality of life. However, in clinical practice most doctors do not usually address patients' spiritual needs and have a limited involvement in their spiritual care. ${ }^{2}$ This aspect of care could be improved by active involvement and participation of local chaplaincy. ${ }^{3}$

We undertook this study to determine awareness of chaplaincy services at our local district general hospital among the junior doctors working in adult medical and oncology wards, and also to assess their views on spiritual support in caring for patients with advanced cancers.

\section{Methods}

Questionnaires were completed by junior doctors to explore their awareness of local chaplaincy services and views on spiritual and religious support in caring patients with advanced cancers. Newly admitted patients with advanced cancers who were either on palliative radio-chemotherapy or supportive management were identified across all general medical wards (excluding cardiology wards, stroke unit and acute admission unit) and the oncology ward over 1 month (1-31 March 2018).

Medical notes were reviewed prospectively to record patients who were offered spiritual and religious support, and also referred to the local chaplaincy services if they wished. Referrals received by the local chaplaincy services from these wards during the study period were also reviewed.

\section{Results and discussion}

There is a lack of awareness of local chaplaincy services among the junior doctors, and spiritual support is perceived as unimportant in caring for patients with advanced cancers.

Thirty-five patients ( 17 male and 18 female) with advanced cancer were admitted over the 1-month period. Thirty-six admissions (17 in oncology, 19 in medical wards) were recorded, with one patient being admitted twice to the oncology ward. Thirty-three of the patients were white British, one was Indian and one was Romanian. Average hospital stay was 13 days.

Authors: Lincoln County Hospital, Lincoln, UK
Patients were admitted for pain control $(n=11)$, weakness, falls and not coping at home $(n=8)$, general deterioration from disease progression ( $=5)$, jaundice $(n=4)$, breathlessness $(n=3)$, nausea and vomiting $(n=1)$, seizures $(n=1)$ and shingles $(n=1)$. Two patients were repatriated to Lincoln following resections of brain tumours.

Twenty-two patients had a religious belief (18 Church of England, one Catholic, one Orthodox, one Spiritualist, one Hindu); 13 were atheists or had no religion. All except the Romanian patient spoke English.

Two patients were deemed to lack mental capacity during admission (one with an advanced brain tumour, one with acute delirium secondary to infection). No one had dementia. Two patients were started on the end-of-life care pathway during admission, and eight were seen by the palliative team while in hospital. Only one patient was documented to have been offered spiritual support by the medical team.

Chaplaincy received 14 referrals from both general medical wards and the oncology ward over the 1-month period. Referrals were mainly made by relatives and nurses, and none by a medical doctor.

\section{Conclusion}

Spiritual support is very occasionally offered by nurses to patients with advanced cancers during their hospital stay. Our study shows that doctors do not routinely offer these patients spiritual support from the local chaplaincy, and we found no difference between the medical and oncology teams. This is due to a significant lack of awareness of local chaplaincy services, and also spiritual support is not viewed as an important aspect of palliative care. Comprehensive palliative care teaching targeting at these areas for local junior doctors should improve the quality of care of patients with advanced cancers.

\section{References}

1 Bai M, Lazenby M. A systematic review of associations between spiritual well-being and quality of life at the scale and factor levels in studies among patients with cancer. J Palliat Med 2015;18:286-98.

2 Smyre CL, Tak HJ, Dang AP, Curlin FA, Yoon JD. Physicians' opinions on engaging patients' religious and spiritual concerns: a national survey. J Pain Symptom Manage 2018;55:897-905.

3 Kernohan WG, Waldron M, McAfee C, Cochrane B, Hasson F. An evidence base for a palliative care chaplaincy service in Northern Ireland. Palliat Med 2007;21:519-25. 